VACCINATION

\title{
bnAbs for HIV: shepherding towards improbable mutations
}

Such
approaches
could also be
applied to the
design of
germline-
targeting
immunogens
for vaccines
to other
pathogens

The discovery of broadly neutralizing antibodies (bnAbs) to HIV in rare HIV-positive individuals indicated that it should be possible to design effective vaccines. However, this has proved to be exceptionally challenging since HIV-targeted bnAbs tend to have many unusual features, including 'improbable mutations' located outside the normal mutation hotspots. Moreover, the inferred precursors of the known bnAbs typically have no detectable affinity for the virus. Therefore, vaccination strategies need to first prime $\mathrm{B}$ cells encoding bnAb precursors, followed by immunogens that shepherd antibody affinity maturation towards the acquisition of unusual features. Two reports in Science now demonstrate the design of immunogens for such a vaccine strategy.

Steichen et al. chose to focus on the bnAb BG18, which, through its heavy chain complementarity-determining region 3 (HCDR3), binds a conserved site on the HIV Env protein and contains improbable mutations. However, it does not have insertions or deletions and may therefore be easier to induce than other bnAbs.

Informed by the crystal structure of BG18 bound to an Env trimer,

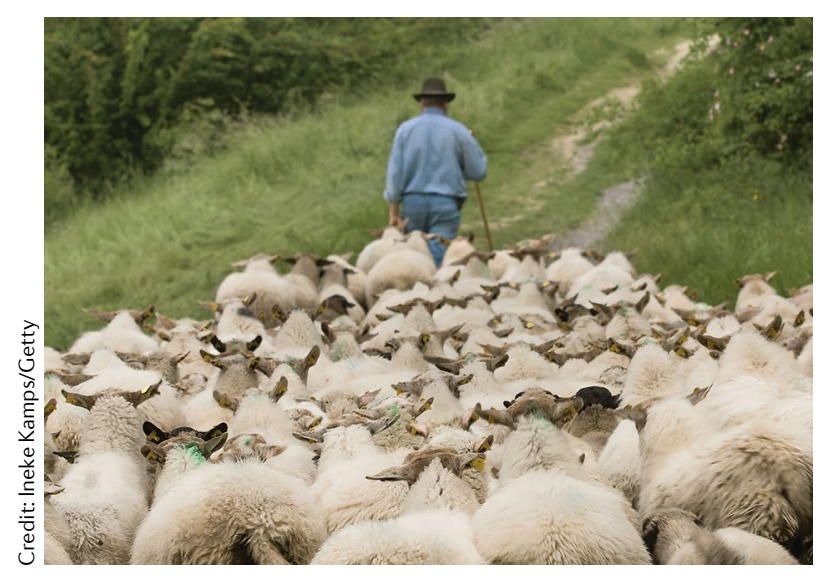

as well as structural and functional information from cryo-electron microscopy (cryo-EM) and mutational studies, the authors were able to define the key genetic features that enable target recognition by BG18. These insights informed a bioinformatics approach to search for potential BG18 precursors in a next-generation sequencing dataset of $>1$ billion sequences of $B$ cell receptor (BCR) heavy chains from $14 \mathrm{HIV}$-negative donors. Encouragingly, precursors were identified in all donors, and the sequence information of their HCDR3 domains was used to design BG18-like precursor antibodies.

Next, these antibodies were used as selection reagents for the directed evolution and optimization of Env trimer immunogens using mammalian cell-surface display libraries. One of the resulting immunogens, the N332-GT2 Env trimer was structurally analysed by cryo-EM. It was found to bind its selecting antibody in a similar manner to binding of the native Env trimer to BG18.

To test whether this immunogen can indeed prime BG18 precursors, the authors generated mice with a very low frequency of BG18 precursorexpressing B cells. They showed that immunization with N332-GT2 trimerbearing nanoparticles can induce sustained germinal centre responses and affinity maturation. Importantly, they also demonstrated that the immunogens can bind to human donor-derived rare naive $\mathrm{B}$ cells that encode BG18-like precursors.

Meanwhile, Saunders et al. hypothesized that immunogens with moderate to high affinity for bnAb precursors and higher affinity for their more mature forms with improbable mutations may both initiate bnAb precursor B cell lineages and guide them towards bnAb development. Focusing on the bnAbs DH270 and CH235, they computationally inferred their clonal history to determine their respective unmutated common ancestors (UCAs). Structural modelling then allowed them to rationally design immunogens that bind to the UCAs, but bind their affinity-matured forms more strongly. Using knock-in mice with B cells that express the variable regions of the respective UCAs, the authors showed that vaccination with a nanoparticle formulation of the immunogens indeed selected for bnAbs with improbable mutations. Cryo-EM structural analysis of induced antibodies demonstrated their similarity to partially matured bnAb precursors.

The antigen designed to induce $\mathrm{CH} 235$-like antibodies was then tested in four outbred rhesus macaques. A course of bimonthly immunizations with the nanoparticle-formulated antigen elicited potent neutralizing antibodies in all animals, and these had similar neutralization signatures to the $\mathrm{CH} 235$-like antibodies early in their bnAb development.

These two recent studies demonstrate approaches to the design of immunogens that bind to bnAb precursors or select for specific improbable antibody mutations. Such approaches could also be applied to the design of germline-targeting immunogens for vaccines to other pathogens. However, the authors note that for the induction of broadly neutralizing polyclonal bnAb responses to HIV, iterative immunogen design will be needed to develop sequential immunogens for the shepherding and polishing of the antibody response.

Alexandra Flemming

ORIGINAL ARTICLES Steichen, J. M. et al. A generalized HIV vaccine design strategy for priming broadly neutralizing antibody responses. Science 366, eaax4380 (2019) | Saunders, K. O. et al. Targeted selection of HIV-specific antibody mutations by engineering $B$ cell maturation. Science 366, eaay7199 (2019) 